\title{
Intravenous gammaglobulin treatment in multiple sclerosis and experimental autoimmune encephalomyelitis: delineation of usage and mode of action
}

\author{
A Achiron, R Gilad, R Margalit, U Gabbay, I Sarova-Pinhas, I R Cohen, E Melamed, \\ O Lider, S Noy, I Ziv
}

\begin{abstract}
Multiple sclerosis (MS) is a central nervous system demyelinating disease of implicated autoimmune aetiology. The effect was evaluated of intravenous gammaglobulin (IVIg), a successful therapy in various autoimmune diseases, in relapsing-remitting $M S$ patients treated for three years. IVIg treatment significantly reduced the number and severity of acute exacerbations and resulted in a lesser neurological disability. There were no significant short or long-term adverse effects to IVIg treatment. To clarify the putative therapeutic effects of IVIg, this treatment was examined in the animal model of experimental autoimmune encephalomyelitis (EAE) in the rat. IVIg suppressed active EAE in relation to disease severity and duration, despite the presence of $T$-cell reactivity to specific antigens, while the treatment had no effect on passive EAE induced by adoptive transfer of myelin basic protein specific CD4 + T-cells. It is concluded that IVIg treatment may be a promising treatment in relapsing-remitting $\mathrm{MS}$ as it can alter the natural course of the disease.
\end{abstract}

Department of

Neurology, Beilinson

Medical Center,

Petah-Tiqva, Israel

A Achiron

R Margalit

U Gabbay

E Melamed

$S$ Noy

I Ziv

Department of

Neurology, Wolfson

Medical Center,

Holon, Israel

R Gilad

I Sarova-Pinhas

Department of Cell

Biology, Weizmann

Institute of Science,

Rehovot, Israel

I R Cohen

O Lider

Correspondence to:

Dr R Gilad, Department of

Neurology, Beilinson

Medical Center

Petah-Tiqva, Israel.

Multiple sclerosis (MS) is the most common central nervous system disease affecting the white matter. Numerous immunological abnormalities have been identified in patients with MS including increased immunoglobureduction in the number of suppressor $T$ cells (CD8) and loss of suppressor function; the presence of activated $\mathrm{T}$ cells (CD4) in the peripheral blood and the migration of inducer to initiate the disease process; increased cytokine production capable of amplifying immune responses and mediating demyelination; enhanced expression of class II major histocompatibility complex (MHC), which results in increased immune reactivity; altered autologous mixed lymphocyte responses on myelin basic protein-coated target cells. ${ }^{1-5}$
The alternations in both cellular and humoral immune mechanisms in MS strongly suggest that immunoregulatory mechanisms mediating suppression are disturbed in the disease. Thus treatment with intravenous gammaglobulins (IVIg) as non-specific modulators of the immune response was suggested as a strategy to alter the grave course of the disease. We have recently reported the beneficial effects of IVIg treatment in relapsingremitting patients with MS. $^{6}$ The present study summarises our long-term clinical experience of IVIg treatment in relapsingremitting MS patients for three years. In addition, we have investigated the mode of action of IVIg in experimental autoimmune encephalomyelitis (EAE), the animal model for MS induced by either active immunisation with myelin-associated proteins or adoptive transfer of syngeneic autoreactive encephalitogenic $\mathrm{T}$ cells. ${ }^{7-10}$

\section{Methods}

\section{CLINICAL STUDIES}

The study was approved by the Medical Ethics Committee on Clinical Investigations of the Beilinson Medical Center, PetahTiqva, Israel. All patients gave informedconsent to participation in the study. lin levels with oligoclonal bands in the CSF; effector cells into the central nervous system

\section{Patients}

Twenty relapsing-remitting MS patients with definite disease, ${ }^{11}$ were included in the study. Selection of patients for participation in the study is described in detail elsewhere. ${ }^{6}$ Briefly, the major inclusion criteria were: age 20-55 years; annual exacerbation rate of at least two well documented exacerbations in the two years before the study; a score no higher than six (ambulatory with assistance) on the Kurtzke Expanded Disability Status Scale (EDSS) ${ }^{12}$; emotionally stable and without severe cognitive impairments; no corticosteroid therapy for at least a month, and/or other immunosuppressive drugs during the previous year before entering the study; negative history of anaphylaxis after previous transfusions of blood or blood products; normal levels of IgA evaluated by plasma immu- 
noelectrophoresis. Randomly, ten patients were assessed to receive IVIg treatment [mean (SD) age 39.8 (10.5); range 21-53] and 10 patients matched for age $[41 \cdot 4(5 \cdot 8)$; range 36-54], disease duration [9.5 (7.3); 8 (4.9), respectively] and annual exacerbation rate in the two years before the study, served as controls.

\section{IVIg treatment}

IVIg (Gamimune N, Miles Inc, Cutter Biological, Promedico, Israel) in a sterile $4.5-5.5 \%$ solution of human protein in 9-11\% maltose, was given intravenously once daily for five consecutive days at a dose of $0.4 \mathrm{~g} / \mathrm{kg} /$ day at the beginning of the study. Thereafter, patients were further treated with booster doses of IVIg, $0.4 \mathrm{~g} / \mathrm{kg} /$ day, once every two months, for the next three years.

\section{Clinical evaluation}

Patients were examined before starting the study, and once every month thereafter. Routine urinalysis, complete blood count and chemistry, serological survey of HTLV-1, HIV, and Hepatitis B, and immunoelectrophoresis were performed at entry to the study and at each six month visit.

\section{Evaluation of acute exacerbations}

Patients were seen at times of suspected exacerbations, that is, when reporting the rapid onset of new symptoms or a deterioration of pre-existing symptoms that persisted for 48 hours or more. An event was counted as an exacerbation only when the patient's symptoms were accompanied by observed objective changes on the neurological examination involving an increase of at least one grade in the score for one of the eight functional groups of the Kurtzke EDSS. Patients experiencing an acute exacerbation were evaluated at frequent intervals, usually every week, until a new, stable neurological base-line is established.

\section{Evaluation of severity of exacerbations}

1) mild-change in one grade in the score for one of the eight functional groups of the Kurtzke EDSS; 2) moderate-change in two grades in the score for one of the eight functional groups of the Kurtzke EDSS or change in one grade in the score for two of the eight functional groups of the Kurtzke EDSS; 3) severe-change in two grades in the score for at least two of the eight functional groups of the Kurtzke EDSS.

\section{Treatment of acute exacerbations}

Severe exacerbations were treated with intravenous methylprednisolone ( $1 \mathrm{gr} / \mathrm{day}$ ) for five consecutive days. Moderate relapses were treated with oral prednisone $(60 \mathrm{mg} /$ day $)$, for five consecutive days. Mild exacerbations were not treated.

\section{EXPERIMENTAL STUDIES}

Animals

Inbred Lewis rats, six to eight weeks old, were supplied by the Animal Breeding Center, Weizmann Institute of Science, Rehovot. Rats were matched for age and sex in each experiment.

\section{Active induction of $E A E$}

Rats were inoculated subcutaneously in each hind footpad with $25 \mu \mathrm{g}$ of guinea-pig myelin basic protein (BP) in complete Freund's adjuvant containing $40 \mu \mathrm{g}$ of Mycobacterium tuberculosis in $0.1 \mathrm{ml}$ of oil.

\section{Passive induction of $E A E$}

Adoptive transfer of EAE was carried out with $2 \times 10^{6}$ activated anti-BP $\mathrm{CD}^{+} \mathrm{K} 1 \mathrm{~T}$ cells per rat, administrated intraperitoneally. ${ }^{7}$

\section{Clinical assessment of $E A E$}

Clinical signs of EAE appeared 11-12 days after active induction and five to seven days after adoptive transfer. Degree of clinical disease was scored as follows: 0-no signs; 1-loss of tail tonicity; 2-paralysis of hind limbs; 3-paralysis of all four limbs; 4 -quadriplegic animal in a moribund state. ${ }^{89}$

\section{IVIg treatment}

Human IgG in a sterile $4 \cdot 5-5 \cdot 5 \%$ solution in 9-11\% maltose, (Gamimune N, Miles Inc., Cutter Biological, Promedico, Israel), was inoculated intravenously into a tail vein at a dose of $0.4 \mathrm{~g} / \mathrm{kg}$ per dose. Controls included bovine serum albumin (BSA), at a dose of $0.4 \mathrm{~g} / \mathrm{kg} /$ day, and Maltose $10 \%, 0.5 \mathrm{ml} /$ day, given intravenously. Treatment was given from the day of disease induction (day 0 ) until clinical signs appeared in the control group.

\section{Reinduction of $E A E$}

Secondary induction of EAE was performed one month after primary immunisation by subcutaneous inoculation in each hind footpad with $25 \mu \mathrm{g}$ of guinea-pig BP in CFA containing $40 \mu \mathrm{g}$ of Mycobacterium tuberculosis in $0.1 \mathrm{ml}$ of oil.

\section{$T$-cell proliferative responses}

Ten days after active induction of EAE lymph node cells were isolated from treated or control rats and incubated in flat-bottomed microtitre plates in quadruplicate wells. Each well contained $2 \times 10^{5}$ cells in $0.2 \mathrm{ml}$ of proliferation medium (Dulbecco's modified Eagle's medium (DMEM), supplemented with $1 \%$ syngeneic fresh rat serum, $5 \times$ $10^{-5} \mathrm{M}$ 2-mercaptoethanol, $2 \mathrm{mM}$ glutamine, $100 \mathrm{U} / \mathrm{ml}$ penicillin and $100 \mathrm{mg} / \mathrm{ml}$ streptomycin), with the following antigens: Mycobacterium tuberculosis, ovalbumin, BP, and the encephalitogenic peptide 71-90, all used at $10 \mu \mathrm{g} / \mathrm{ml}$; Con-A, $2.5 \mu \mathrm{g} / \mathrm{ml}$; BSA, 20 $\mu \mathrm{g} / \mathrm{ml}$. After 72 hours of incubation at $37^{\circ} \mathrm{C}$ in a humidified atmosphere plus $10 \% \mathrm{CO}_{2}$, each well was pulsed with $1 \mu \mathrm{Ci}$ of $\left[{ }^{3} \mathrm{H}\right]$ thymidine for 16 hours. The cultures were then harvested on fibreglass filters and thymidine incorporation was measured using a liquid scintillation counter. The proliferative response was expressed as cpm (SD). 
Figure 1 Effect of IVIg on $M S$ exacerbation rate.

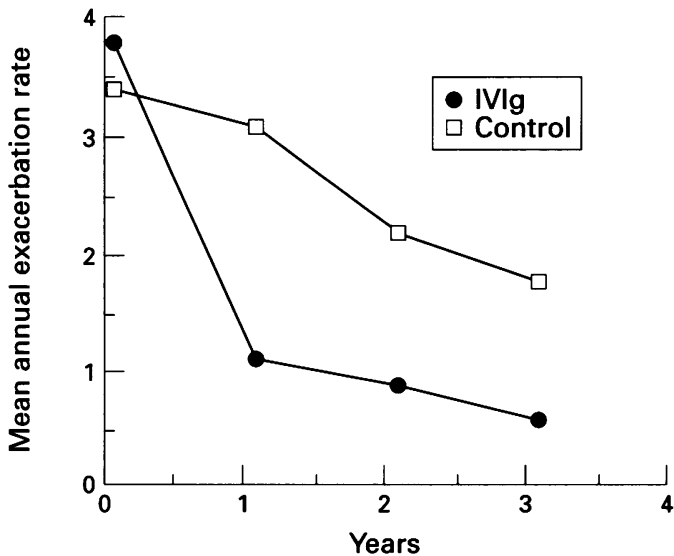

Statistical analysis

Student's $t$ test, Chi-square and Spearman's correlation test were used to evaluate differences between the various groups.

\section{Results}

Effect of IVIg on MS acute exacerbations

The major effect of IVIg on exacerbation rate was observed after the first year of treatment (fig 1, table 1). The mean (SD) annual exacerbation rate (AER) decreased from $3.7(1 \cdot 2)$ at baseline to $1(0.7)(p<0.001)$ after one year of IVIg treatment, while in the control group AER before the study remained unchanged after one year [3.3 (1.4) and 3.0

Figure 2 Effect of IVIg treatment on severity of acute exacerbations.

Figure 3 Effect of IVIg treatment on the EDSS.
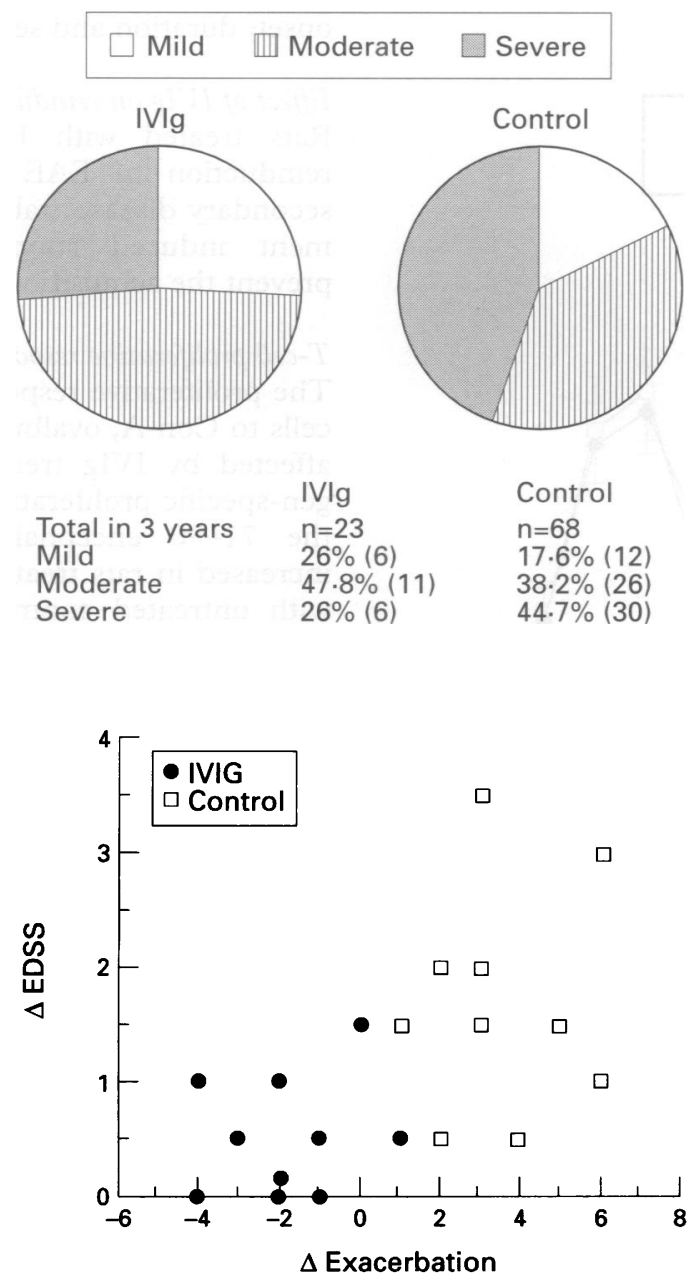

Table 1 Effect of IVIg on acute exacerbations

\begin{tabular}{|c|c|c|c|}
\hline Exacerbation rate & $I V I g$ & Control & Pvalue \\
\hline $\begin{array}{l}\text { Before treatment } \\
\text { Mean (SD) }\end{array}$ & $3 \cdot 7(1 \cdot 4)$ & $3 \cdot 3(1 \cdot 2)^{\star}$ & 0.512 \\
\hline $\begin{array}{l}\text { 1) year } \\
\text { Mean (SD) }\end{array}$ & $1.0(0 \cdot 7)$ & $3.0(1.6)$ & 0.002 \\
\hline 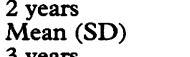 & $0.8(0.8)$ & $2 \cdot 1(1)$ & 0.005 \\
\hline $\begin{array}{l}3 \text { years } \\
\text { Mean (SD) }\end{array}$ & $0.5(0.5)$ & $1 \cdot 7(0 \cdot 7)$ & 0.001 \\
\hline
\end{tabular}

(1.6) respectively, $p=0 \cdot 279$ ]. The AER was similar in the two groups before treatment ( $p$ $=0.512$ ); however the decrease in the number of acute exacerbations in the IVIg treated patients, resulted in a significant difference of the AER $(p=0.002)$ between the groups after one year. During the second and third years of the study, IVIg treated MS patients had significantly less exacerbations than untreated patients. The mean (SD) AER in the IVIg group after the second year was 0.8 $(0 \cdot 8)$ and in the control untreated patients $2 \cdot 1$ (1), $p=0.005$. The mean (SD) AER in the IVIg group after the third year of treatment was $0.5(0.5)$ and $1.7(0.7)$ in the control MS patients $(p<0.001)$. The annual exacerbation rate was inversely correlated to duration of IVIg treatment $(\mathrm{r}=0.74, \mathrm{p}=$ 0.0002 ), and to the neurological disability after three years $(r=0.7, p=0.0005)$.

Severity of acute exacerbations was also influenced by IVIg treatment (fig 2). The majority of exacerbations in the IVIg treated patients were mild to moderate, while in untreated control patients most of the acute exacerbations were moderate to severe (Chisquare $=14 \cdot 06, \mathrm{p}=0.007)$.

Effect of IVIg treatment on neurological disability The mean EDSS score showed no significant change by IVIg treatment, although the numbers were slightly higher in the control untreated group at one and three years compared with baseline. The mean (SD) change in EDSS (AEDSS) calculated per patient showed a trend of lessened disability in the IVIg group at the one year endpoint [ $\triangle$ EDSS $=-0.3(0.58)]$ compared with the control group [ $\triangle \mathrm{EDSS}=0.2(1 \cdot 0), \mathrm{p}=0 \cdot 182]$. At the three year endpoint the $\triangle E D S S$ became significant between the two groups; in the IVIg group $\triangle \mathrm{EDSS}$ was $0.35(0.58)$, compared with $1.7(0.98)$ in the control untreated patients $(p=0.001)$.

Correlation between the change in acute exacerbations and neurological disability from baseline to the end of three years in each patient, clearly showed that in IVIg treated MS patients the reduction in the number of acute exacerbations was correlated to a lesser neurological disability, while the opposite occurred in the untreated control group (fig 3).

Effect of IVIg treatment on actively induced EAE In IVIg treated animals, only $70 \%$ of rats developed EAE with no mortality, while in untreated rats all animals developed EAE with a mortality rate of $30 \%$ (table 2 , fig 4 ). 
Table 2 Effect of IVIg treatment on actively induced EAE

Figure 4 Effect of IVIg treatment on the development of experimental autoimmune encephalomyelitis in rats.

Figure 5 Effect of IVIg treatment on the development of passively induced experimental autoimmune encephalomyelitis in rats.

\begin{tabular}{|c|c|c|c|c|c|c|}
\hline \multirow[b]{2}{*}{ Treatment } & \multicolumn{3}{|c|}{ Evaluation of clinical symptoms of $E A E$} & \multirow[b]{2}{*}{ Duration (days) } & \multirow[b]{2}{*}{$\begin{array}{l}\text { Maximal (SD) } \\
\text { EAE score }\end{array}$} & \multirow{2}{*}{$\frac{\text { Reinduction }}{\% \text { Incidence }}$} \\
\hline & $\begin{array}{l}\text { \% Incidence } \\
\text { (No of rats) }\end{array}$ & $\%$ Mortality & Onset (day) & & & \\
\hline $\begin{array}{l}\text { None } \\
\text { Maltose } 10 \% \\
\text { BSA } \\
\text { IVIg }\end{array}$ & $\begin{array}{l}100(40) \\
100(10) \\
100(10) \\
70(40)\end{array}$ & $\begin{array}{r}30 \\
20 \\
0 \\
0\end{array}$ & $\begin{array}{l}12 \\
11 \\
12 \\
12\end{array}$ & $\begin{array}{l}9 \\
9 \\
9 \\
7\end{array}$ & $\begin{array}{l}3.0(1.4) \\
2.9(1.5)^{\mathrm{b}} \\
2.8(1.2)^{\mathrm{b}} \\
1.6(0.98)^{\mathrm{a}}\end{array}$ & $\begin{array}{l}0 / 5 \\
N D \\
N D \\
0 / 5\end{array}$ \\
\hline
\end{tabular}

Following induction of EAE, rats were either untreated or treated with IVIg, $10 \%$ maltose or BSA. Reinduction was done one month after primary induction of EAE.

${ }^{\mathrm{a}} \mathrm{p}-\mathrm{p}<0.05 v$ untreated group.

bp-not significant.

ND-not determined.
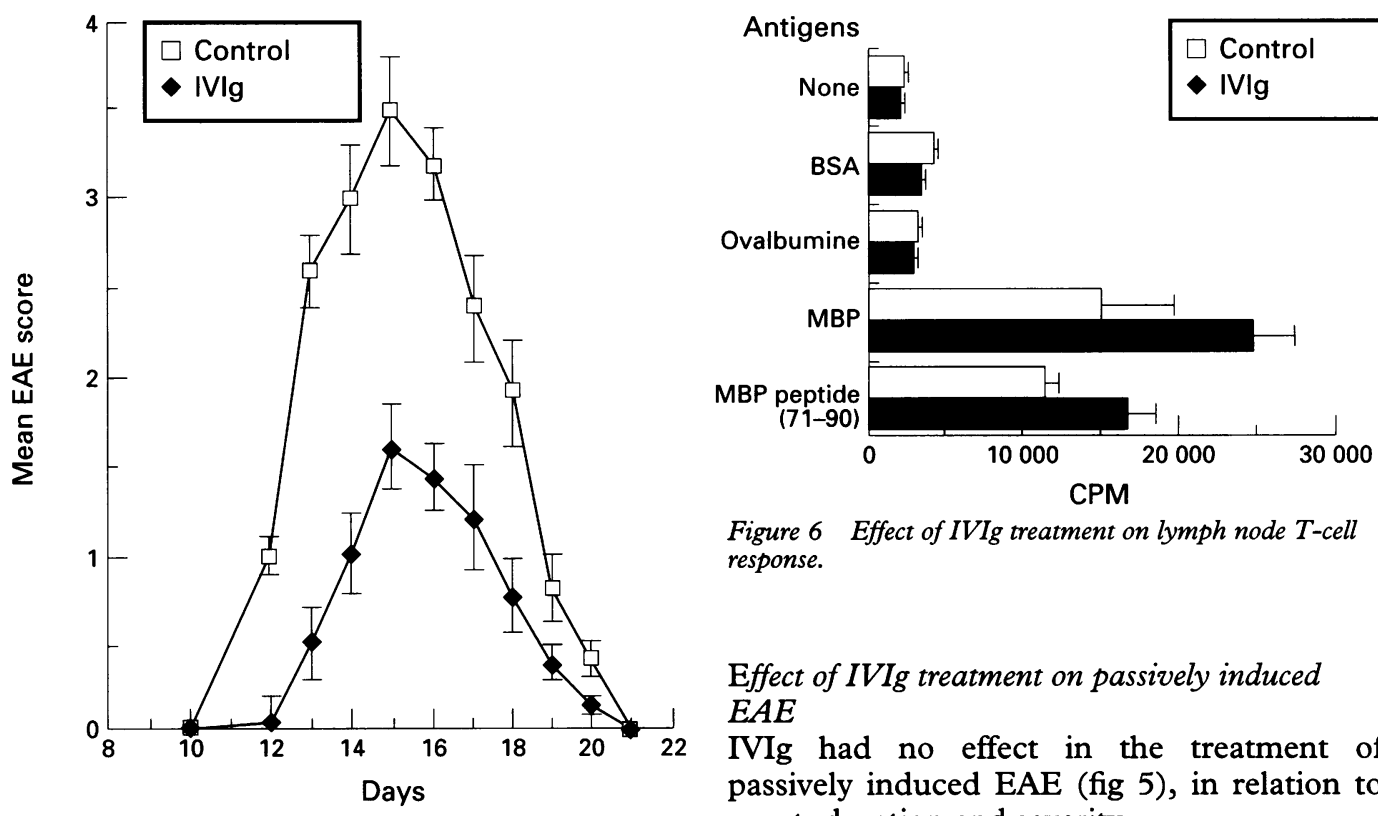

Figure 6 Effect of IVIg treatment on lymph node T-cell response.

Effect of IVIg treatment on passively induced $E A E$

IVIg had no effect in the treatment of passively induced EAE (fig 5), in relation to onset, duration and severity.

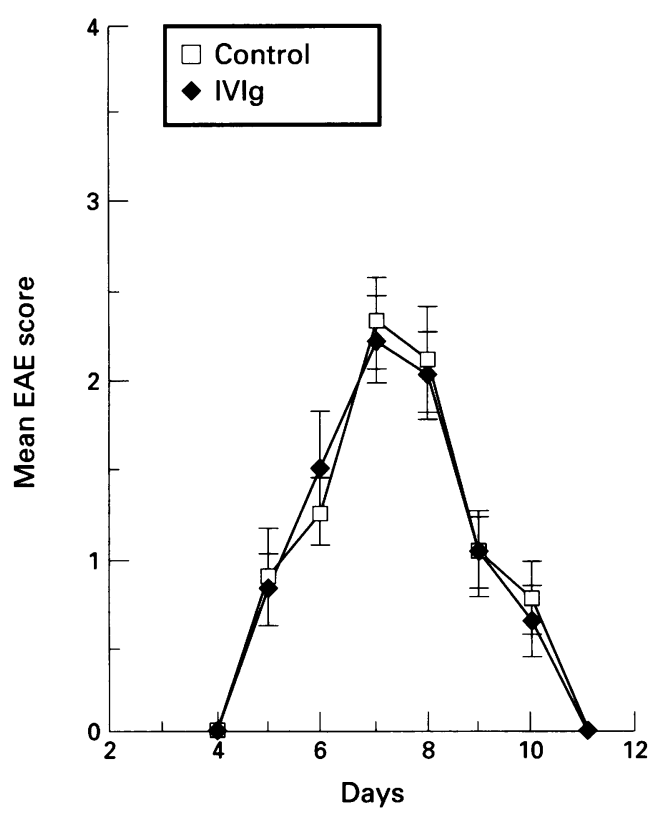

In the IVIg treated rats EAE was mild. The mean maximal EAE score was 1.6 compared with 3.0 in untreated animals $(p<0.05)$. Duration of EAE was shorter in the IVIg treated rats, seven days compared to nine days in untreated animals. Onset of EAE was not affected by IVIg treatment. The severity of disease in MBP-immunised rats treated with $10 \%$ maltose or BSA was similar to the results of untreated animals.
Effect of IVIg on reinduction of EAE

Rats treated with IVIg were resistant to secondary disease (table 2). Thus IVIg treatment induced suppression but did not prevent the acquisition of resistance.

\section{$T$-cell proliferative responses}

The proliferative responses of lymph node $T$ cells to Con-A, ovalbumin and BSA were not affected by IVIg treatment. However, antigen-specific proliferative responses to $\mathrm{BP}$ and the 71-90 encephalitogenic peptide were increased in rats treated with IVIg compared with untreated controls (fig 6). Thus EAE suppression by IVIg treatment was associated with a strong $\mathrm{T}$-cell response to the target encephalitogenic peptides.

\section{Discussion}

The results of this study indicate that IVIg is well tolerated and has beneficial effects on the course of relapsing-remitting MS. Over a period of three year treatment, IVIg had significant effects on exacerbation rates, severity of exacerbations, and neurological disability. The major reduction in exacerbation rate was observed after the first year of IVIg treatment, and persisted during the next two years of therapy. The high exacerbation rate in the patients with MS that entered this study, enabled effective evaluation of IVIg treatreinduction of EAE and did not develop 
ment, in spite of the small number of patients included. The decrease in exacerbation rate in the untreated group may be related to the natural course of the disease, reduced stress as a result of participation in a study protocol, or corticosteroid therapy during acute moderate and severe exacerbations. After three years the reduced exacerbation rate in IVIg treated MS patients was correlated with lesser neurological disability, while untreated patients had more exacerbations and increased EDSS score. The beneficial effects of IVIg were also evident in the animal model of EAE. IVIg suppressed active induction of EAE, while it had no effect on passively induced disease. Thus it appears that IVIg can alter immune response before $T$-cell activation. The results of increased proliferative $T$-cell responses to target antigens along with active EAE suppression suggest that the mechanism by which IVIg alter the natural course of the disease is not through inhibition of T-cell proliferation. This is further supported by the results of secondary EAE induction, where no disease developed, implying that IVIg suppression of primary induction had no effect on the acquisition of resistance.

In conclusion, the use of IVIg in relapsingremitting $M S$ has a significant beneficial effect on the natural course of the disease over time. Though the mechanism by which
IVIg modulates the immune system is not yet clear, it probably involves $T$-cell interactions during the active phase of the disease.

1 Waksman BH, Reynolds WE. Multiple sclerosis as a disease of immune regulation. Proc Soc Exp Biol Med 1984;175:282-94

2 Golaz J, Steck A, Moretta L. Activated T lymphocytes in patients with multiple sclerosis. Neurology 1983;33: 1371-3.

3 Reder AT, Arnason BGW. Immunology of multiple sclerosis. In: PJ Vinken, GW Bruyn, HC Klawans, eds. Handbook of clinical neurology. Amsterdam; Elsevier, 1985;47:337-96.

4 Antel JP, Reder AT, Noronha AB. Cellular immunity and immune regulation in multiple sclerosis. Semin Neurol 1985;5:117-26.

5 Noronha A, Toscas A, Jensen MA. Interferon beta augments suppressor cell function in multiple sclerosis. $A n n$ Neurol 1990;27:207-10.

6 Achiron A, Pras E, Gilad R, Ziv I, Mendel M, Gordon CR, Noy S, Sarova-Pinhas I, Melamed E. Open controlled therapeutic trial of high-dose intravenous immunoglobulins in relapsing-remitting multiple scleroimmunoglobulins in relapsing-remit

7 Mor F, Cohen IR. T cells in the lesion of experimental autoimmune encephalomyelitis. Enrichment for reactivities to myelin basic protein and heat shock proteins. $\mathcal{J}$ Clin Invest 1992;90:2447-55.

8 Mokhatarian VF, McFarlin DE, Raine CS. Adoptive transfer of myelin basic protein-synsitized $T$ cells produce chronic relapsing demyelinating disease in mice. Nature 1984;309:356-8.

9 Lemire JM, Weigle WO. Myelin basic protein-specific T cell clones and experimental allergic encephalomyelitis. Pathol Immunopathol Res 1986;5:248-52.

10 Zamvil SS, Steinman L. The T lymphocyte in experimental allergic encephalomyelitis. Annu Rev Immunol 1990; tal allergic enc.

11 Poser CM, Paty DW, Scheinberg L, et al. New diagnostic criteria for multiple sclerosis: guidelines for research protocols. Ann Neurol 1983;13:227-31.

12 Kurtzke JF. Rating neurologic impairment in multiple sclerosis: an expanded disability status scale (EDSS). Neurology 1983;33:1444-52. 